\title{
PANCREATIC ADENOCARCINOMA WITH PENILE METASTASIS
}

\author{
Waqas Jehangir, Julie I Woods, Zankhana Mehta, Mellar P. Davis \\ Department of Palliative Medicine, Geisinger Medical Centre, Danville, PA, USA \\ Received: 20 July 2017 / Accepted 10 September 2017
}

A 79-year-old male admitted for poor oral intake, lack of appetite, $15 \mathrm{lbs}$ weight loss and cramping lower abdominal pain. Computed tomography abdomen/pelvis was consistent with pancreatitis. He was treated with intravenous fluids. Given the absence of alcohol abuse, Ca 19-9 was obtained which was elevated at 6041 . Gastroenterology team was consulted and an oesophagogastroduodenostomy and endocscopic ultrasound was performed which showed a hypoechoic, heterogeneous mass in the pancreatic tail. Fineneedle aspiration pathology revealed adenocarcinoma of the pancreas [Figure 1], and staging scans revealed metastasis to the kidney. He started therapy with gemcitabine and paclitaxel and radiation therapy.

Three months later, the patient reported lumps on his penis and discomfort when retracting his foreskin. He subsequently noticed a lump on the head of his penis and multiple lumps underneath the skin on the shaft of the penis. Genitalia examination showed an uncircumcised penis, and numerous diffuse subcutaneous hard lesions of the shaft of penis measuring from $2 \mathrm{~mm}$ to $1 \mathrm{~cm}$ were noted. There was a 3-mm reddened lesion on the head of the penis. Nodules were also noted on the shaft and deep base of the penis. An magnetic resonance imaging showed the largest mass involving the corpus cavernosum at the base of the penis, with the two largest lesions measuring $3 \mathrm{~cm} \times 1.9 \mathrm{~cm}$ on the left and $2 \mathrm{~cm} \times 1.4 \mathrm{~cm}$ on the right [Figures 2 and 3]. 3 weeks later, he developed urinary obstruction, and he underwent urethral dilation and cystoscopy; with placement of a suprapubic urinary catheter. Biopsy showed metastatic adenocarcinoma to the penis [Figure 4]. The patient was offered palliative radiation to the penis but refused. He was discharged home with hospice where he died 2 weeks later.

Penile metastases are exceedingly rare, only a few hundred cases are described in the literature and most originate from either genitourinary primary or colonic primaries. ${ }^{[1]}$ To the

$\overline{\text { Correspondence: Dr. Waqas Jehangir, Palliative Medicine, Geisinger }}$ Medical Centre, Danville, PA, USA.

Email: wjehangir@hotmail.com

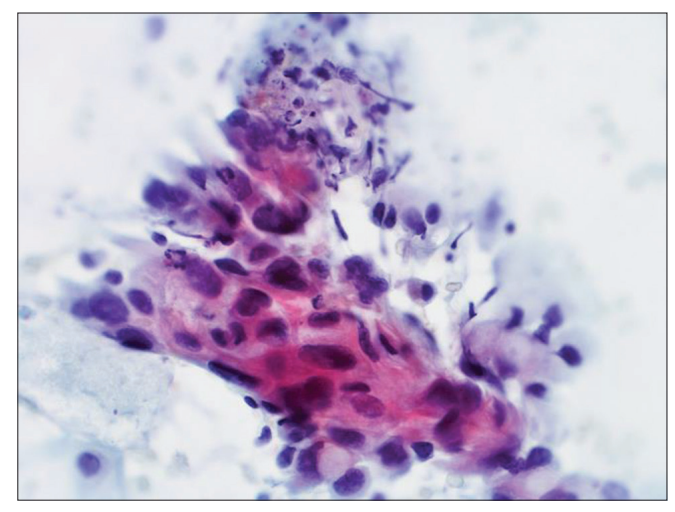

Figure 1: Adenocarcinoma of the pancreas

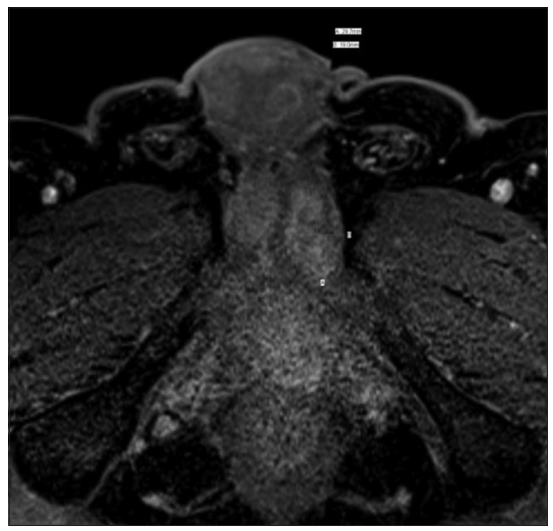

Figure 2: Magnetic resonance imaging - Left corpus cavernosum lesion



Figure 3: Magnetic resonance imaging - Right corpus cavernosum lesion 


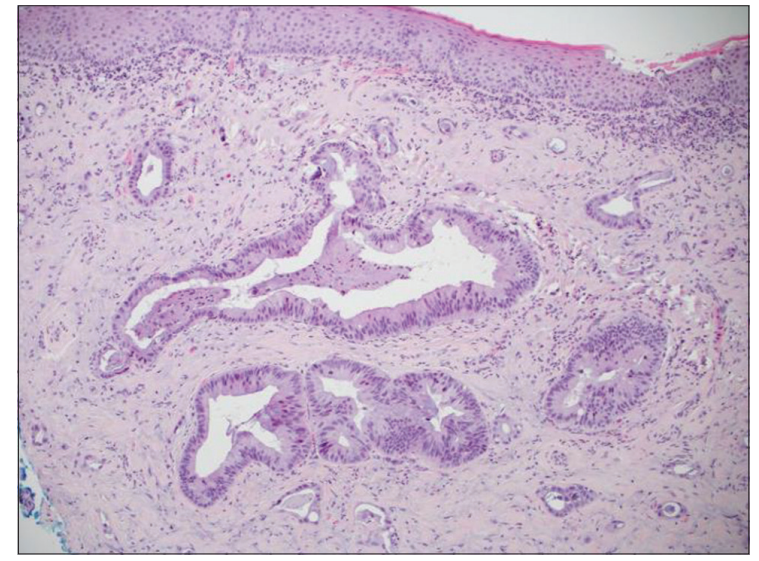

Figure 4: Metastatic adenocarcinoma to the penis

best of our knowledge, this is the third case reported in the USA. The most common metastasis of pancreatic cancer are to the liver, regional lymph nodes, peritoneal cavity and lung and less commonly to the brain, bone and adrenal glands. ${ }^{[2]}$

Most common metastatic mechanism is hematogenous spread through the portal venous system to the liver. ${ }^{[3]}$ Diagnosis is usually made by biopsy or fineneedle aspiration which differentiates primary from metastasis. Treatment options include radical or partial penectomy, local excision, hormonal or chemotherapy depending on the primary stage, and radiation therapy for palliation. Our patient experience is that penile metastasis though rare can present with masses and cause urinary obstruction.

\section{Conflict of Interest}

The authors declare that they have no conflict of interest.

\section{References}

1. Harper M, Arya M, Shah PJ. A lump in the penis. J R Soc Med 2002;95:38-9.

2. Blastik M, Plavecz E, Zalatnai A. Pancreatic carcinomas in a 60-year, institute-based autopsy material with special emphasis of metastatic pattern. Pancreas 2011;40:478-80.

3. Kamisawa $\mathrm{T}$, Isawa $\mathrm{T}$, Koike $\mathrm{M}$, et al. Hematogenous metastases of pancreatic ductal carcinoma. Pancreas 1995;11:345-9. 